\title{
Multicomponent Alginate-Derived Hydrogel Microspheres Presenting Hybrid Ionic-Covalent Network and Drug Eluting Properties
}

\author{
Luca Szabó, François Noverraz, Sandrine Gerber-Lemaire* \\ Institute of Chemical Sciences and Engineering, Group for Functionalized Biomaterials, Ecole Polytechnique Fédérale de Lausanne, \\ EPFL SB ISIC SCI-SB-SG, Station 6, CH-1015 Lausanne, Switzerland, Sandrine.Gerber@epfl.ch \\ Dedicated to Prof. Michael Graetzel at the occasion of his $75^{\text {th }}$ birthday
}

\begin{abstract}
The development of multifunctional encapsulation biomaterials could help the translation of cell-based therapies into standard medical care. One of the major hurdles in the field of encapsulated cell transplantation is the current lack of materials presenting optimal properties, including long term stability, mechanical durability and non-immunogenic character. Modification of sodium alginate ( $\mathrm{Na}$-alg) with polyethylene glycol (PEG) derivatives, without restricting its gelling abilities, appeared as an efficient strategy to produce dual ionic-covalent spherical hydrogels with enhanced mechanical performance as well as drug-eluting microspheres (MS) for the mitigation of inflammatory response after transplantation. In this study, the combination of PEGylated alginates equipped with cross-reactive functionalities and the anti-inflammatory drug ketoprofen (KET) resulted in the assembly of multifunctional (MF) hybrid MS, merging the advantages of ionic-covalent hydrogels with the ability for controlled drug delivery. Physical characterization confirmed their improved mechanical resistance, their higher shape recovery performance and increased stability toward non-gelling ions, as compared to pure Ca-alg hydrogels. In vitro release kinetics revealed the controlled and sustained delivery of KET for over two weeks.
\end{abstract}

Keywords: alginate $\bullet$ microspheres $\bullet$ ketoprofen $\bullet$ controlled drug release $\bullet$ cross reactive polymers $\bullet$ multifunctional hydrogel

\section{Introduction}

Cell-based therapies recently emerged as a promising alternative to treat many disorders, where the replacement or substitution of dysfunctional or damaged cells with functional substitutes can treat or even cure the diseases. ${ }^{\left[{ }^{2]}\right.} \mathrm{A}$ variety of medical fields could profit from cell-based therapies, including diabetes treatment, liver regeneration, hematology, cancer treatment, and vascular diseases, amongst others. ${ }^{[2]}$ Several FDA approved cell-therapy products indicate the real potential of this approach, such as Provenge for the treatment of prostate cancer, ${ }^{[3]}$ or the two CAR (chimeric antigen receptors) T-cell products Kymriah and Yescarta. ${ }^{[4]}$ Encapsulation of the therapeutic cells within a $3 \mathrm{D}$ membrane prior to transplantation not only provides a protection from the host immune system, but further broadens the cell sources from only allogeneic to xenogeneic derived donors, offering the perspective for an inexhaustible cell supply. In addition, the necessity of immunosuppressive treatment, which comes along with many undesirable side effects, for both the patient and the transplanted cells, could be reduced or completely avoided. Since the introduction of the term "artificial cell" by Chang in $1964,{ }^{[5]}$ many efforts have been put toward developing encapsulation materials with adequate properties for high cell survival and long-term graft functionality. ${ }^{[6-8]}$ The encapsulating matrix should ensure fast bidirectional diffusion of oxygen, nutrients, therapeutic compounds and metabolic products, while not inducing a severe immune response. High mechanical durability and elasticity of the cell-surrounding environment is also a prerequisite for a successful encapsulation. ${ }^{[6]}$

Hydrogels, due to their large water content, are highly biocompatible and have the potential of mimicking extracellular matrix like properties. ${ }^{[9]}$ The natural polysaccharide alginate (alg) is the most widely used biomaterial for cell immobilization owing to its ability for fast hydrogel microsphere (MS) formation through ionic gelation in the presence of divalent cations $\left(\mathrm{Ca}^{2+}, \mathrm{Mg}^{2+}\right.$, etc) and its high cell compatibility. ${ }^{[10]}$ The presence, on the alg backbone, of hydroxyl and carboxyl groups amenable for chemical functionalization makes it a versatile material for human and xenogeneic cell transplantation applications. ${ }^{[12]} \mathrm{A}$ few clinical trials addressed the transplantation of cells enveloped in alg-based materials, with limited success. ${ }^{[12-14]}$ Despite great promise, the translation to a common clinical use is still awaited. In spite of the relative simplicity of the cell encapsulation concept, the complexity of the practical implementation poses numerous challenges. Two of the main limitations for a safe and efficient therapy based on encapsulated cells include the poor mechanical properties of alg-based materials due to the instability of the ionic network and adverse immune reaction of the host system in the form of pericapsular fibrotic overgrowth (PFO).

To improve long-term MS integrity, mechanical durability and chemical stability, polyelectrolyte complexation of the polyanionic alg core with polycations such as poly-L-lysine (PLL), poly-L-ornithine (PLO), poly-L-arginine (PLA) or chitosan, was proposed. Human islet containing Alg-PLO-alg MS improved mean daily blood glucose levels and decreased exogenous insulin necessity following transplantation into diabetic patients. However, long-term graft functionality could not be achieved. ${ }^{[15,16]} \mathrm{A} 30 \%$ reduction in the insulin necessity of a diabetic patient was achieved with transplantation of fetal porcine islets in alg-PLL-alg MS, but only for a period less than a year-long. ${ }^{[7]}$ Besides, the biocompatibility issues related to the use of polycation layers require 


\section{HELVETICA}

alternative approaches. ${ }^{[18,19]}$ The reinforcement of alg hydrogel ionic network was achieved by combination with poly(ethylene glycol) (PEG) ${ }^{[20,22]}$ or UVinduced photo-cross-linking in the presence of methacrylate residues. ${ }^{[22-24]}$ However, depending on the application, the exposure to UV light might be detrimental to cells. A more cell-friendly approach is the use of click chemistry for the covalent cross-linking of alg derivatives containing cross-reactive functionalities such as furan-maleimide, ${ }^{[20,21]}$ tetrazine-norbornene, ${ }^{[22]}$ azide-alkyne, ${ }^{[23]}$ or thiol-thiol. ${ }^{[24-26]}$ Most of these derivatives were produced by the functionalization of the alg carboxyl moieties, thus reducing the density of available sites for ionic gelation.

Many parameters can trigger a foreign body response (including size, morphology and purity of materials) by launching a series of complex inflammatory responses and leading to a fibrotic tissue development around the microcapsules that blocks the diffusion of essential small molecules. ${ }^{[27]}$ Many parameters can trigger a foreign body response (including size, morphology and purity of materials) by inducing a series of complex inflammatory events and leading to the development of fibrotic tissue around the MS that blocks the diffusion of essential small molecules and invariably leads to cell necrosis. ${ }^{[27]}$ One of the numerous strategies that have been proposed to mitigate fibrotic reactions targets the surface of alg-MS by reducing its adhesive properties to prevent the adsorption of macrophages and proteins. Coating of alg MS with chitosan ${ }^{[33]}$ or PEG ${ }^{[28,29]}$ showed reduction in the PFO and decrease of protein surface adsorption. More recently, surface modification of alg MS with polymer brushes was proposed to mitigate the formation of fibrotic tissues. ${ }^{[30,31]}$ Another approach aimed at reducing the severity of inflammatory responses by the co-encapsulation of mesenchymal stem cell $s^{[32,33]}$ or regulatory T cells $s^{[34,35]}$ with islets, which delayed graft rejection due to reduced fibrosis in both allo- and xenotransplantation models. Alternatively, the incorporation of small antiinflammatory or anti-fibrotic drugs within the hydrogel matrix was investigated for further delivery at the transplantation site. However, due to the porous structure of alg-based hydrogels, premature burst release of the drug was often reported. ${ }^{\left[{ }^{[36]}\right.}$ Co-encapsulation of curcumin, ${ }^{[37]}$ pentoxifylline ${ }^{[38]}$ and $\mathrm{KET}^{[39]}$ were explored in the context of alg-based islet transplantation, and led to decreased PFO. Though the drug release was not maintained for a prolonged time. Using the inclusion ability of cyclodextrin (CD), Omtvedt et al. proposed the combination of native alg with CD-conjugated oxidized alg for the prolonged delivery of methyl orange, as drug model. ${ }^{\left[4^{0}\right]}$ However, the stability of the resulting MS was highly compromised by the presence of $C D$ units and the oxidation of the alg backbone.

We previously presented the development of dual ionic-covalent alg-derived hydrogels based on the covalent conjugation of the alg hydroxyl groups with cross-reactive heterobifunctional PEG derivatives. The resulting MS were formed by fast ionic gelation, following extrusion into a $\mathrm{Ca}^{2+}$-containing bath, and reinforced by slower spontaneous formation of a covalent network, without the need for external chemical cross-linker. ${ }^{[4]]}$ This strategy resulted in significant stabilization of the hydrogel MS in conditions which lead to complete liquefaction of purely ionic Ca-alg MS, as well as improved shape recovery performance and high potential for the microencapsulation of endocrine cells. Following a similar strategy, PEGylated ketoprofen was conjugated to the matrix of alg MS, allowing sustained release of the anti-inflammatory drug, in vitro and in vivo. ${ }^{[42]}$ The slow release of ketoprofen, controlled by the hydrolysis rate of the conjugation linkage, resulted in the clear mitigation of PFO following the transplantation of encapsulated insulin producing MIN6 cells in immune competent mice.

Herein, we present the development of alg-based multifunctional (MF)-MS which combine a dual ionic-covalent matrix with drug-eluting properties, with the aim to address severe issues in the field of encapsulated cell transplantation, which are the lack of durability of the hydrogel network and the incidence of PFO. First, we synthesized PEGylated alg derivatives, that contain functionalities for covalent cross-linking via 1,4-addition or ketoprofen conjugated through a hydrolysable ester linkage. These alg derivatives were then combined to produce hydrogel MF-MS, which were characterized for their mechanical performance and drug eluting ability.

\section{Results and Discussion}

\section{Preparation of Alg-PEG derivatives}

For the formation of MF-MS, we envisaged the combination of alg-derivatives that are able to: i) produce spherical hydrogels assembled by a dual network of ionic interactions and covalent bonds resulting from 1,4-addition of thiol to carbon electrophiles; and ii) release anti-inflammatory drugs via controlled hydrolytic reaction. To achieve the formation of such systems, Alg-SH, Alg-ACR, Alg-ACA and Alg-KET polymers (Figure 1) were synthesized according to functionalization protocols previously established in our group. ${ }^{[26,41,42]} \mathrm{Na}$-alg was first converted into the corresponding tetrabutylammonium (TBA) salt, allowing further selective activation of the hydroxyl groups and condensation with heterobifunctional PEG derivatives (for detailed synthesis protocols, see Supplementary Material S-3-S-6). Alg functionalization with cross-reactive PEG chains was targeted at a grafting degree that does not interfere with the ionic gelation properties of alg, and allows the formation of spherical hydrogel MS by simple extrusion of solutions of complementary Alg-PEG polymers into a gelation bath containing divalent cations $\left(\mathrm{Ca}^{2+}\right)$. We found that a grafting degree between 13 and $30 \%$ for the cross-reactive functionalities aligned with these expectations. Based on ${ }^{1} \mathrm{H}-\mathrm{NMR}$ analysis $(800 \mathrm{MHz})$, the grafting degree of Alg-PEG derivatives was assessed by comparison of integration of ${ }^{1} \mathrm{H}$ resonances of alginate unit and characteristic PEG moieties. In case of overlapping signals, separation was achieved by deconvolution with Lorentzian functions (for detailed protocol, see Supplementary Material S-10). ${ }^{[41,42]}$ 

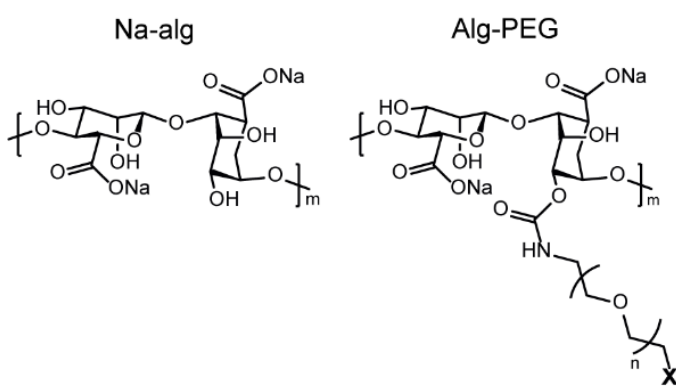

\begin{tabular}{c|c|c|c|c}
$\mathbf{X}$ & $\mathrm{n}$ & Name & Grafting (\%) & Function \\
\hline $\mathrm{SH}$ & $=44$ & Alg-SH & $29^{\mathrm{a}}$ & covalent cross-linking \\
& $=44$ & Alg-ACR & $26^{\mathrm{a}}$ & covalent cross-linking \\
& $=22$ & Alg-ACA & $13^{\mathrm{b}}$ & covalent cross-linking \\
\end{tabular}

Figure 1. Structures of Na-alg and synthesized Alg-PEG derivatives for the formation of MF-MS. aDetermination of the grafting degree was achieved by deconvolution with Lorentzian functions (Supplementary Material, Figure S9). ${ }^{b}$ Determination of the grafting degree was achieved by direct comparison of integration of ${ }^{1 \mathrm{H}}$ resonances.

The purity and chemical structure of Alg-PEG polymers were confirmed by analysis of their ${ }^{1} \mathrm{H}-\mathrm{NMR}$ spectra. The characteristic peaks related to the specific cross-reactive end functionalities confirmed the successful conjugation of PEG derivatives to the alg backbone. Figure 2 represents sections of the ${ }^{1} \mathrm{H}-\mathrm{NMR}$ spectra with the characteristic signals of the PEG end-functionalities: 3.23 (t) ppm for the $\mathrm{CH}_{2}-\mathrm{SH}$ (Alg-SH); $6.33-6.19$ (m) and 5.79 (dd) for the olefinic protons of acrylamide (Alg-ACA); 6.47 (dd), 6.24 (dd) and 6.02 (dd) ppm for the olefinic protons of acrylate (Alg-ACR); and 7.85-7.58 (m) for the aromatic protons and 1.53 (d) ppm for the methyl protons of ketoprofen (Alg-KET). Further evidence for the covalent attachment to the alg backbone was established by diffusion ordered spectroscopy (DOSY) (for representative DOSY spectrum, see Supplementary Materials S-9, Figure S8).
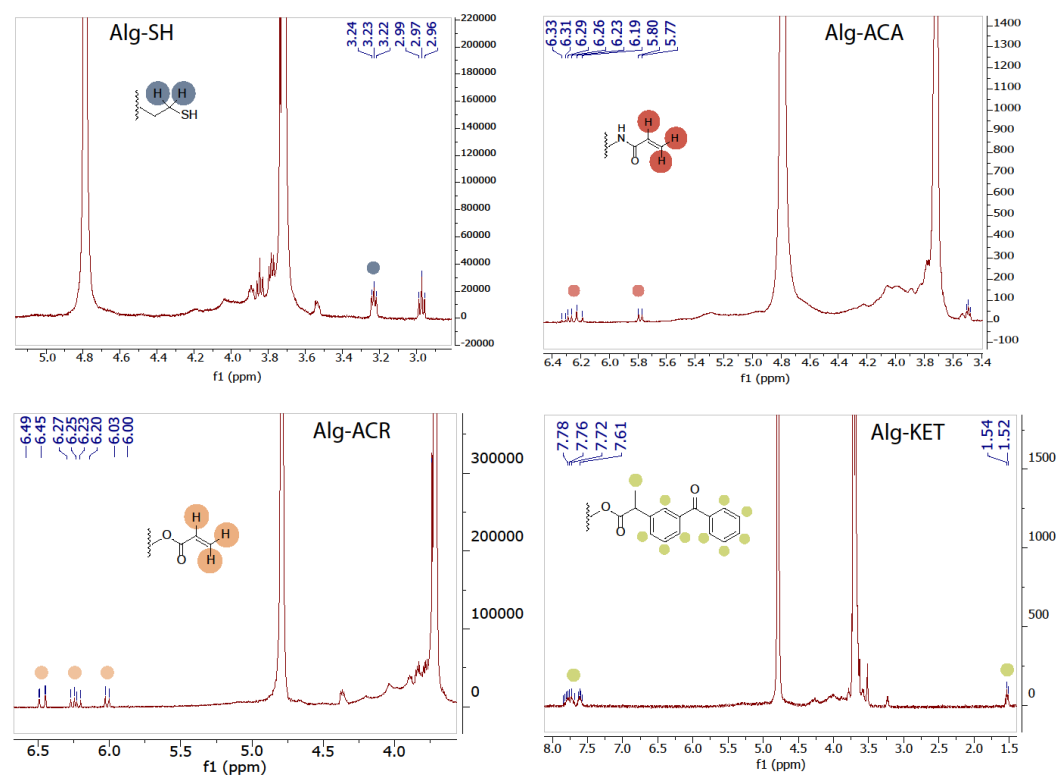

Figure 2. ${ }^{1} \mathrm{H}-\mathrm{NMR}$ spectra with the characteristic peaks belonging to Alg-SH, Alg-ACA, Alg-ACR and Alg-KET.

\section{Preparation of MF-MS and their characterization.}

MF-MS of two different compositions were produced from the combination of cross-reactive Alg-PEG derivatives with Alg-KET, which contains an ester bond for slow hydrolysis under physiological conditions. We previously established the efficient reinforcement of alg-based MS by combination of crossreactive PEGylated alginate derivatives, giving rise to fast ionic interactions with calcium ions and slower covalent sulfur-carbon bond formation. ${ }^{[41]}$ In the present study, we proposed to investigate the effect of the linkage to the PEG chain (ester in Alg-ACR vs amide in Alg-ACA) on the physical properties and drug eluting profile of the resulting MF-MS. Preliminary assays demonstrated the need to add Na-alg to the multicomponent system in order to ensure sphericity of the produced hydrogels. MF-MS (ACR) were thus produced by mixing Alg-ACR, Alg-SH, Alg-KET and Na-alg, while MF-MS (ACA) resulted from the combination of Alg-ACA, Alg-SH, Alg-KET and Na-alg in a 1:1:1:1 weight ratio. First, Alg-PEG polymers were dissolved separately (2 to 3 wt\%) in MOPS buffer (10 mM, pH=7.4) containing $0.4 \% \mathrm{NaCl}$. Upon complete dissolution, the four polymer components were mixed together until homogenization. The resulting mix-polymers solution was extruded into a gelation bath containing $\mathrm{Ca}^{2+}$ ions, using a co-axial airflow droplet generator, for concomitant formation of ionic interactions and covalent sulfur-carbon bonds (Figure 3). 
a)

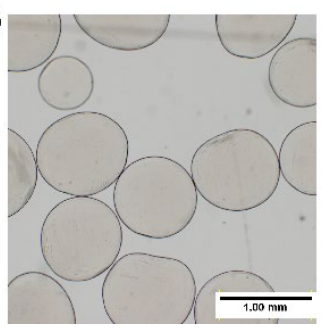

b)

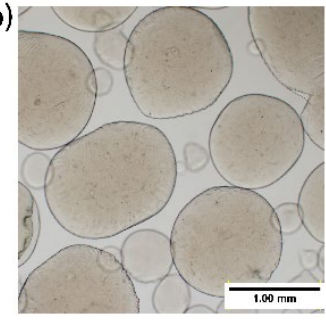

c)

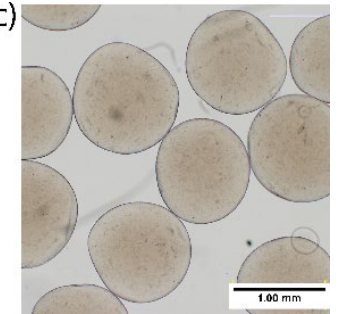

Figure 3. Bright-field microscopy images of a) Ca-alg MS b) MF-MS (ACR) and c) MF-MS (ACA). Images were taken on the day of MS formation (scale bar 1.o mm).

MF-MS were characterized for their diameter, mechanical resistance to uniaxial compression, shape recovery performance upon multiple successive compressions, stability towards non-gelling ions, permeability to different sizes of dextran molecules and for their ketoprofen release profile. Pure Ca-alg MS (2 wt\%) were prepared under the same conditions for comparison. The diameter and resistance of the beads were assessed after their production (day o), and followed at day 3 and day 7 after preparation to figure the evolution of the hydrogel network (Table 1). A gradual decrease of the size of the MS was observed over one week, more noticeably for the MF-MS. While the size and mechanical resistance of Ca-alg MS did not show much variation over time, both MF-MS underwent significant shrinking, in agreement with the increase of chemical cross-linking density as the covalent network is slowly forming (from $1.21 \mathrm{~mm}$ at day o to $0.94 \mathrm{~mm}$ at day 7 for MF-MS (ACR) and from $1.11 \mathrm{~mm}$ at day o to $0.99 \mathrm{~mm}$ at day 7 for MF-MS (ACA)). This observation was accompanied by a remarkable increase in the resistance to 9o\% compression of their initial diameter. While Ca-alg MS displayed almost no change in resistance to compression from day o $\left(1.68 \mathrm{~N} / \mathrm{mm}^{3}\right)$ to day $7\left(1.70 \mathrm{~N} / \mathrm{mm}^{3}\right)$, MF-MS increased their resistance to compression by a factor of 4.15 for MF-MS (ACR) (from 0.72 at day o to $2.99 \mathrm{~N} / \mathrm{mm}^{3}$ at day 7 ) and of 1.95 for MF-MS (ACA) (1.14 at day o to $2.22 \mathrm{~N} / \mathrm{mm}^{3}$ at day 7 ). This increasing mechanical resistance is attributed to the slow formation of covalent cross-links over time, and correlates with the decreasing MS diameter. MS were assessed for their mechanical properties at day 11 and 14 after bead formation, but no more enhancement was observed at these time points (data not shown). Therefore, we concluded that formation of the dual cross-linked hydrogel network was completed within one week, and further characterization of MF-MS was thus performed after one week.

Table 1. Main diameter and mechanical resistance to uniaxial compression of MS over one week.

\begin{tabular}{lllll}
\hline Time & Properties & Ca-alg & MF-MS (ACR) & MF-MS (ACA) \\
\hline Day o & $\mathrm{d}_{0}{ }^{\mathrm{a}}(\mu \mathrm{m})$ & $959 \pm 80$ & $1212 \pm 133$ & $1111 \pm 199$ \\
& Resistance $^{\mathrm{b}}\left(\mathrm{N} / \mathrm{mm}^{3}\right)$ & $1.68 \pm 0.18$ & $0.72 \pm 0.33$ & $1.14 \pm 0.18$ \\
Day 3 & $\mathrm{d}_{3}{ }^{\mathrm{a}}(\mu \mathrm{m})$ & $931 \pm 115$ & $1039 \pm 133$ & $1052 \pm 189$ \\
& Resistance $\left(\mathrm{N} / \mathrm{mm}^{3}\right)$ & $1.45 \pm 0.15$ & $1.70 \pm 0.29$ & $1.76 \pm 0.18$ \\
Day 7 & $\mathrm{d}_{7}{ }^{\mathrm{a}}(\mu \mathrm{m})$ & $923 \pm 103$ & $943 \pm 129$ & $989 \pm 92$ \\
& Resistance $^{\mathrm{b}}\left(\mathrm{N} / \mathrm{mm}^{3}\right)$ & $1.70 \pm 0.18$ & $2.99 \pm 0.27$ & $2.22 \pm 0.14$ \\
\hline
\end{tabular}

aMeasured on a Leica DM 5500 microscope, 30 MS/condition. bMeasured on a Texture Analyzer, using 10 MS/condition.

In view of the future targeted application of the MS in cell-based therapy, a good shape recovery ability is a prerequisite for withstanding the whole process of transplantation and subsequent potential mechanical impacts in vivo over a prolonged time. We assessed the shape recovery performance of Ca-alg and MF-MS at day 0, 3 and 7 post bead generation, by compressing the MS to $90 \%$ of their initial diameter and repeating the compression 10 consecutive times. While unmodified Ca-alg MS has been shown to suffer from poor elastic properties, ${ }^{[26,43]}$ PEGylation and the presence of covalent cross-links prompted an enhanced shape recovery ability of MF-MS. As a consequence of the slowly evolving covalent network over one week, the elastic properties were gradually increasing at each time point of the measurement, reaching around 50\% shape recovery by day 7 . Under the same conditions, Ca-alg MS recovered only $30 \%$ of their initial shape by day 7 . 
A)

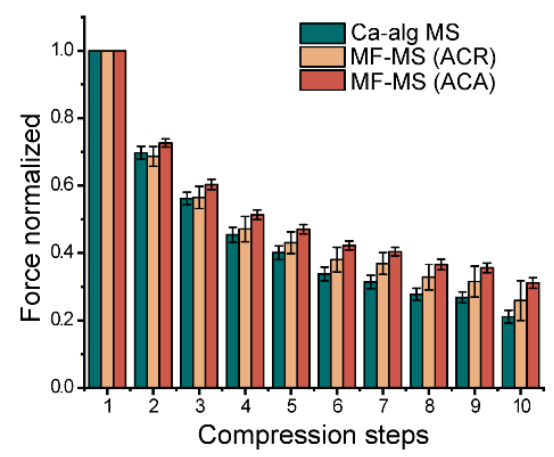

B)

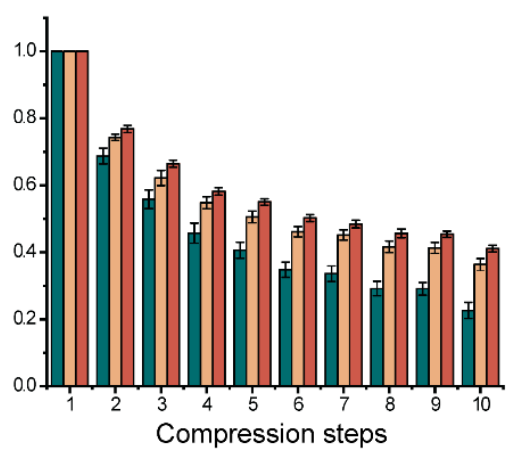

C)

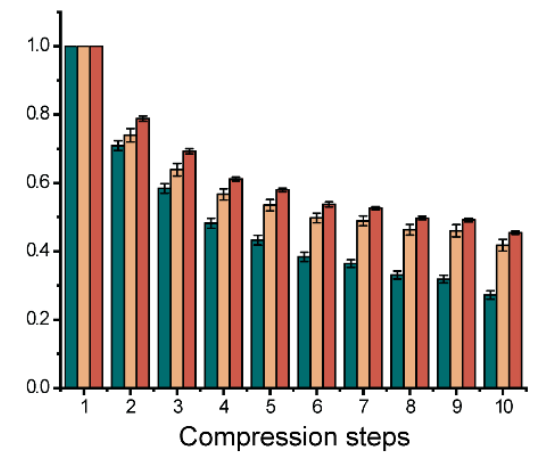

Figure 4. Shape recovery performance after 10 consecutive compressions to $90 \%$ of the initial diameter of Ca-alg MS, MF-MS (ACR) and MF-MS (ACA) at A) day o; B) day 3; and C) day 7 after MS formation. Error bars represent the standard deviation from $\mathrm{n}=10$ measurements.

Ca-alg MS are known to swell under physiological conditions, ${ }^{[44]}$ due to the presence of non-gelling ions. This is one of the main factors which triggers the decrease of MS integrity in vivo, and eventually leads to the complete liquefaction of the beads, discontinuing the protection of the enveloped cells. We tested the stability of MF-MS towards increasing concentrations of Na-citrate solutions, which functions as a chelating agent and provides a non-gelling ion environment. Table 2 summarizes the effect of increasing concentrations of Na-citrate on the integrity of Ca-alg MS and MF-MS (Supplementary Material S-12, Figure S11). MF-MS of both compositions showed enhanced stability compared to Ca-alg MS. While we experienced a negligible extent of swelling when increasing the concentration of chelator, due to the exchange of $\mathrm{Ca}^{2+}$ to $\mathrm{Na}^{+}$, the presence of covalent cross-linkings contributed to maintain the stability and sphericity of MF-MS. MF-MS (ACA) displayed complete integrity, and minimal swelling up to 20 mM Na-citrate concentration. MF-MS (ACR) were stable up to $30 \mathrm{mM}$ concentrations, ensuring integrity and sphericity of the MS. Surprisingly, the presence of acrylamide did not result in increased stability compared to acrylate functionality, indicating that the length of grafted PEG unit has a larger influence on the swelling behavior of the MS than the chemical functionality itself (PEG-ACR MM=2000 $\mathrm{g} \mathrm{mol}^{-1}, \mathrm{PEG}-\mathrm{ACA} M M=1000 \mathrm{~g} \mathrm{~mol}^{-1}$ ). Nonetheless, both MF-MS maintained a spherical shape up to the limit of dissolution, unlike Ca-alg which suffered from partial MS breakage from low concentration of Na-citrate. This emphasizes the important contribution of the covalent cross-linkings in enhancing MS stability in the presence of non-gelling ions and chelating agents.

Table 2. Stability of MS upon exposure to increasing concentration of Na-citrate solution. ${ }^{\mathrm{a}}$

\begin{tabular}{|c|c|c|c|c|c|}
\hline & $5 \mathrm{mM}$ & $10 \mathrm{mM}$ & $20 \mathrm{mM}$ & $30 \mathrm{mM}$ & $40 \mathrm{mM}$ \\
\hline Ca-alg MS & Stable & Stable & Partially dissolved & Dissolved & Dissolved \\
\hline MF-MS (ACR) & Stable & Stable & Stable & Stable & Dissolved \\
\hline MF-MS (ACA) & Stable & Stable & Stable & Dissolved & Dissolved \\
\hline
\end{tabular}

aMS were immersed in aqueous solutions of Na-citrate for $24 \mathrm{~h}$. Stability was assessed by observation of the MS under a Leica DM 5500 microscope.

The permeability profile of MF-MS, in comparison with Ca-alg MS, was established by incubation under gentle stirring in solutions of fluorescein isothiocyanate (FITC)-dextran standards of increasing molecular weights ( 40 to $500 \mathrm{kDa}$ ) in buffered solutions (MOPS, $10 \mathrm{mM}$, pH = 7.4). After 36 hours, the percentage of infiltration was quantified on cross-sectional confocal images (Supplementary Material S-11, Figure S10) and expressed as the ratio of fluorescence intensity (pixel quantification with ImageJ) within and outside the MS, using glass beads as a reference (Figure 5). The observed permeability values were similar to previously published data for Ca-alg MS, ${ }^{[45,46]}$ and confirmed that the density of PEG chains did not significantly alter the diffusion profile of the resulting hydrogels, especially in the case of MF-MS (ACA). Hence, we concluded that grafting of PEG derivatives and subsequent covalent cross-linking can be added as a reinforcement of alg-based MS network while maintaining the favorable permeability profile of native Ca-alg hydrogels. 


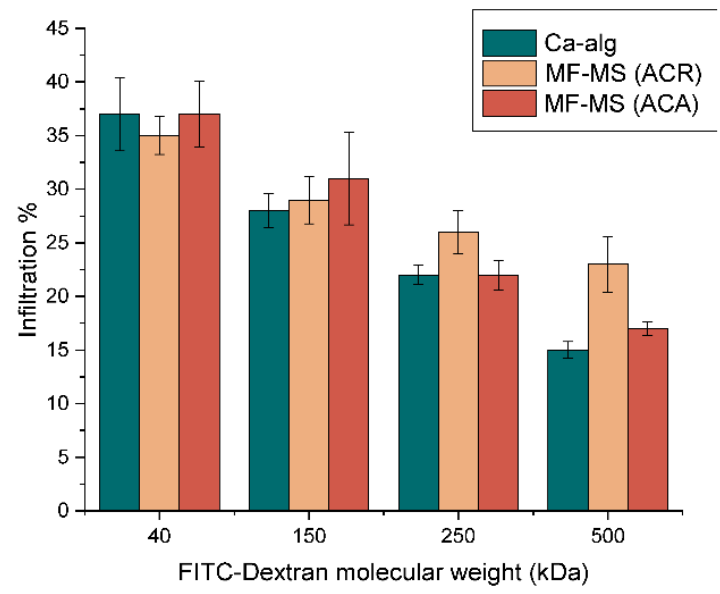

Figure 5. Permeability of Ca-alg, MF-MS (ACR) and MF-MS (ACA) towards solutions of FITC-labeled dextran of 40, 150, 250 and $500 \mathrm{kDa}$ molecular weight. Quantification was performed using Image J after $36 \mathrm{~h}$ of incubation. Error bars represent the standard deviation from $\mathrm{n}=3$ measurements, using glass beads as reference.

\section{Drug eluting properties of MF-MS.}

The release of KET was monitored over two weeks in MOPS solution ( $3 \mathrm{~mL}, 10 \mathrm{mM}, \mathrm{pH}=7.4$ ) containing $\mathrm{CaCl}_{2}$ (100 mM). The cumulative release profile was established from LC-MS quantification in aliquots collected from the supernatant at regular time points (Figure 6). MF-MS of both compositions displayed a controlled pattern, with no sign of burst release, allowing sustained delivery of KET up to $408 \mathrm{~h}$ after MS formation. These results suggest that the nature of the electrophilic Alg-PEG partner implied in the covalent cross-linking (acrylate vs acrylamide) does not impact the drug eluting properties of the multicomponent hydrogels. As MF-MS are simply assembled by combination of cross-reactive and drug-functionalized Alg-PEG derivatives, the methodology herein presented offers a versatile approach to the production of encapsulation materials with tunable physical properties and equipped for mitigation of PFO by slow release of anti-inflammatory compounds at the site of transplantation.

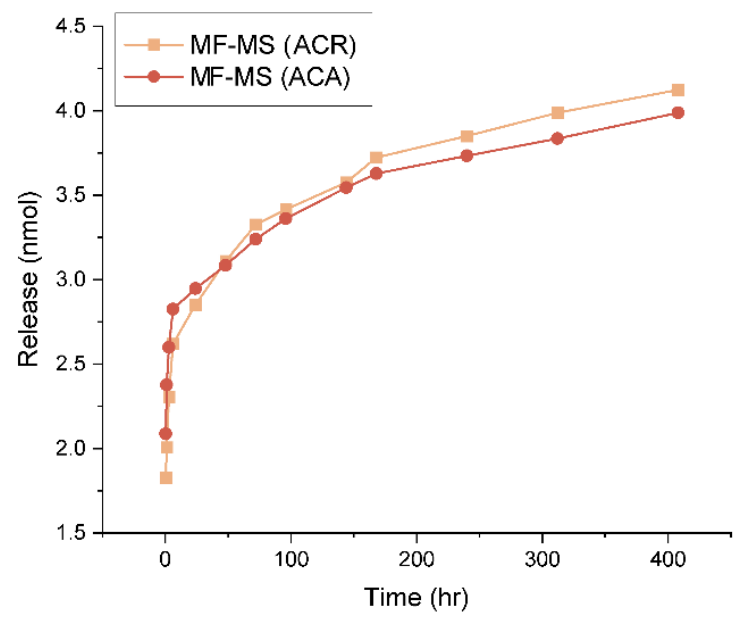

Figure 6. In vitro cumulative release of ketoprofen from MF-MS (ACR) and MF-MS (ACA). MS were stored in MOPS solution ( $3 \mathrm{~mL}, 10 \mathrm{mM}, \mathrm{pH}=7.4$ ) containing CaCl2 (100 mM), under gentle agitation. Aliquots of the supernatant were withdrawn at regular time points and analyzed by LC-MS (Supplementary Material S-13). At each sampling time, fresh medium was added to compensate for the withdrawn volume.

\section{Conclusions}

Despite the immense promise of encapsulated cell based-therapies, translation to clinical applications is still hampered by the lack of proper encapsulation biomaterials fulfilling the many requirements for long-term in vivo graft functionality. The outcome of cell transplantation highly depends on the stability and durability of the material, as well as on the extent of PFO induced. We herein described the development of a polymeric matrix that outperform some of the limitations of alg hydrogels, which still represent the most valuable leads for clinical transplantation of encapsulated endocrine cells. PEGylated alg polymers were prepared and their grafting degrees were adjusted to the need of spherical hydrogel production. Combination of these derivatives, equipped with cross-reactive end functionalities and the anti-inflammatory drug KET, resulted in the formation of dual ionic-covalent and drug-eluting MF-MS by 


\section{HELVETICA}

simple extrusion into a gelation bath containing $\mathrm{Ca}^{2+}$ ions. Mechanical properties of MF-MS, in particular their resistance to uniaxial compression and their elasticity under repeated stress, showed remarkable improvement compared to pure Ca-alg MS, due to the presence of covalent cross-linking. Additionally, MF-MS (ACR) presented higher stability towards a non-gelling environment than only physically cross-linked Ca-alg MS, maintaining spherical shape and integrity under conditions which led to complete liquefaction of purely ionic MS. Finally, the excellent drug eluting properties of MF-MS via slow ester hydrolysis suggests that this polymeric assembly could be an attractive candidate for cell encapsulation applications, where there is a need for combined durability and mitigation of adverse PFO.

\section{Experimental Section}

\section{Materials and Methods}

Na-alg Kelton HV (lot no. $61650 \mathrm{~A},[\eta]=813 \mathrm{~mL} \mathrm{~g}^{-1}$ in $0.1 \mathrm{M} \mathrm{NaCl}, \mathrm{T}=25^{\circ} \mathrm{C}, \mathrm{G} / \mathrm{M}=0.6$ ) was obtained from Kelco (San Diego, USA, CA). Linear PEG starting materials were purchased from Biochempeg (PEG-ACR, MM $=2000 \mathrm{~g} \cdot \mathrm{mol}^{-1}$, PEG-ACA, MM $\left.=1000 \mathrm{~g} \cdot \mathrm{mol}^{-1}\right)$, Sigma $\left(P E G-S H, M M=2000 \mathrm{~g} \cdot \mathrm{mol}^{-1}\right)$ and Jenkem Technology (BocNH-PEG-OH, MM = $2000 \mathrm{~g} \cdot \mathrm{mol}^{-1}$ ). Other reagent-grade solvents (Fluka, Riedel-de-Haën) and chemicals (Aldrich, Acros, Fluka, Sigma, Maybridge, TCI Chemicals, Apollo and Fluorochem) were used without further purification. NMR spectra were recorded on Bruker Avance III-400, Bruker Avance-400 or Bruker DRX-400 spectrometers (Bruker, Billerica, MA, USA) at room temperature (rt), unless otherwise stated. ${ }^{1} \mathrm{H}$ frequency is at $400.13 \mathrm{MHz},{ }^{13} \mathrm{C}$ frequency is at $100.62 \mathrm{MHz}$. Chemical shifts are reported downfield from tetramethylsilane. ${ }^{1} \mathrm{H}$ and ${ }^{13} \mathrm{C}$ signals are reported in ppm and the resonance multiplicity is described as s (singlet), d (doublet), t (triplet), m (multiplet). Coupling constants ( $)$ are given in hertz (Hz). Solvent used for NMR spectroscopy was deuterated water $\left(\mathrm{D}_{2} \mathrm{O}\right.$, Sigma-Aldrich).

\section{Synthesis and characterization protocols}

Functionalization of Na-alg with PEG derivatives. PEGylated alg polymers were prepared according to previously reported protocols.[41,42] Detailed procedure is given in the Supplementary Material S-3-S-6. Alg-SH ${ }^{1} \mathrm{H}-\mathrm{NMR}\left(400 \mathrm{MHz}\right.$, Deuterium oxide): 4.19-3.52 (m, $\left.\mathrm{CH}_{-} \mathrm{Alg}+\mathrm{CH}_{2}-\mathrm{CH}_{2}-\mathrm{O}\right), 3.23(t, \mathrm{~J}=$ $\left.5.0 \mathrm{~Hz}, 2 \mathrm{H}, \mathrm{CH}_{2}-\mathrm{SH}\right), 2.97\left(t, \mathrm{~J}=6.1 \mathrm{~Hz}, 2 \mathrm{H}, \mathrm{CH}_{2}-\mathrm{NH}-\mathrm{C}(\mathrm{O}) \mathrm{O}\right)$. Alg-ACR ${ }^{1} \mathrm{H}-\mathrm{NMR}(400 \mathrm{MHz}$, Deuterium oxide): $6.47(d d, \mathrm{~J}=17.3,1.0 \mathrm{~Hz}, 1 \mathrm{H}, \mathrm{CH}=\mathrm{CH}-\mathrm{O}), 6.24$ $\left(d d, \mathrm{~J}=17.3,10.5 \mathrm{~Hz}, 1 \mathrm{H}, \mathrm{CH}_{2}=\mathrm{CH}-\mathrm{O}\right), 6.02\left(d d, \mathrm{~J}=10.7,0.9 \mathrm{~Hz}, 1 \mathrm{H}, \mathrm{CH}_{2}=\mathrm{CH}-\mathrm{O}\right), 4.35-4.34\left(m, 2 \mathrm{H}, \mathrm{CH}_{2}-\mathrm{O}-\mathrm{C}(\mathrm{O})\right), 3.88-3.52\left(m, \mathrm{CH}_{-} \mathrm{Alg}+\mathrm{CH}_{2}-\mathrm{CH}_{2}-\mathrm{O}\right), 2.94$ $\left(t, J=4.9 \mathrm{~Hz}, 2 \mathrm{H}, \mathrm{CH}_{2}-\mathrm{NH}\right)$. Alg-ACA ${ }^{1 \mathrm{H}-N M R}\left(400 \mathrm{MHz}\right.$, Deuterium oxide): $6.33-6.19\left(m_{1}, 2 \mathrm{H}, \mathrm{CH}_{2}-\mathrm{CH}-\mathrm{C}(\mathrm{O})+\mathrm{CH}_{2}-\mathrm{CH}-\mathrm{C}(\mathrm{O})\right) 5.79(d d, \mathrm{~J}=9.9,1.6 \mathrm{~Hz}, 1 \mathrm{H}$, $\left.\mathrm{CH}_{2}=\mathrm{CH}-(\mathrm{O})\right), 4.22-3.67\left(m, \mathrm{CH}-\mathrm{Alg}+\mathrm{CH}_{2}-\mathrm{CH}_{2}-\mathrm{O}\right), 3.49\left(t, \mathrm{~J}=5.2 \mathrm{~Hz}, 2 \mathrm{H}, \mathrm{CH}_{2}-\mathrm{NH}-\mathrm{C}(\mathrm{O})-\mathrm{O}\right), 3.23\left(m_{1} \mathrm{CH}_{2}-\mathrm{PEG}\right)$. Alg-KET ${ }^{1} \mathrm{H}-\mathrm{NMR}$ (400 MHz, Deuterium oxide): $7.85-7.58(m, \mathrm{Ar}-\mathrm{H}), 4.26-3.50\left(m, \mathrm{CH}-\mathrm{Alg}+\mathrm{CH}_{2}-\mathrm{CH}_{2}-\mathrm{O}\right), 3.23\left(m_{1} \mathrm{CH}_{2}-\mathrm{NH}-\mathrm{C}(\mathrm{O})\right), 1.53\left(d, \mathrm{~J}=7.2 \mathrm{~Hz}, \mathrm{CH}_{3}\right)$. For ${ }^{1} \mathrm{H}-\mathrm{NMR}$ spectra, see Supplementary Material, S-7-S-8.

\section{Formation of MF-MS}

Alg-PEG polymers were dissolved in $10 \mathrm{mM}$ MOPS buffer, $\mathrm{pH} 7.4$, containing $0.4 \% \mathrm{NaCl}$, at the indicated concentration. The polymer solutions were mixed in equivalent weight ratios and the resulting solutions were gently agitated for 5 to 15 minutes to ensure homogeneous distribution. The solution of mixed polymers was extruded into a gelation bath (100 $\mathrm{mM} \mathrm{CaCl}_{2}$ in $10 \mathrm{mM} \mathrm{MOPS} \mathrm{buffer,} \mathrm{pH} 7.4$ ) containing tween 80 (1/10 000). MS were produced employing a coaxial air-flow droplet generator. MS were recovered by filtration $(70 \mu \mathrm{m})$, and rinsed with the gelation solution without tween 80 . MS were kept in the gelation bath (100 $\mathrm{mM} \mathrm{CaCl}_{2}$ in $10 \mathrm{mM}$ MOPS buffer, $\mathrm{pH}_{7.4}$ ), until further characterization or drug release quantification.

\section{Physical characterization of MF-MS}

The average diameter (thirty MS of each polymer were included in the analysis) of the MS was measured on a Leica DM 5500 microscope equipped with a DMC 2900 color camera. The mechanical resistance to $90 \%$ uniaxial compression of the initial MS diameter was analyzed using a texture analyzer (TA-XT2i, software Texture Exponent 32, Stable Micro Systems, Godalming, UK) equipped with a force transducer (1 mN resolution). A single MS was placed below the probe, for which a constant speed was set at $0.5 \mathrm{~mm} \mathrm{~s}^{-1}$. To obtain the shape recovery (elasticity) profile, the same compression to $90 \%$ of the initial diameter was repeated 10 consecutive times on one MS. Ten MS of each polymer were included in the analysis.

\section{Permeability measurements}

Ca-alg, MF-MS (ACR), MF-MS (ACA) and control glass beads were incubated for $36 \mathrm{~h}$ with fluorescein isothiocyanate (FITC)-labeled dextran solution (0.033 $\mathrm{mg} / \mathrm{mL}$, in MOPS buffer, $\mathrm{pH} 7.4$ ) of 40, 150, 250 and $500 \mathrm{kDa}$ molecular weights. The MS were then imaged with a confocal microscope (LSM 700, Zeiss). Permeability of FITC-dextrans within the MS was quantified using ImageJ software, on 3 hydrogel MS/condition and 3 glass beads/condition. The infiltration 
ratio is given as a percentage, considering the glass beads o\% as a reference. For representative confocal images, see Supplementary Material S-11, Figure S10.

\section{Stability towards Na-citrate solution}

Ca-alg, MF-MS (ACR) and MF-MS (ACA) stored in MOPS solution (10 mM MOPS buffer, $\mathrm{pH}=7.4$, containing $100 \mathrm{mM} \mathrm{CaCl}_{2}$ ) were collected by filtration, dried and placed in $1 \mathrm{~mL}$ of $\mathrm{Na}$-citrate solutions (prepared in MOPS buffer, $\mathrm{pH}=7.4$ ) of 5, 10, 20, 30 and $40 \mathrm{mM}$, for $24 \mathrm{~h}$. The MS were then imaged using an Olympus AX70 microscope equipped with an Olympus DP70 color digital camera. For microscope images, see Supplementary Material S-12, Figure S11.

\section{Quantification of ketoprofen release}

MF-MS (ACR) and MF-MS (ACA) prepared from $1 \mathrm{~mL}$ of mixed polymers solution were placed in $3 \mathrm{~mL}$ of $\mathrm{MOPS}$ medium (10 $\mathrm{mM}$, containing $100 \mathrm{mM}$ CaCl $\mathrm{m}_{2}$ at $\mathrm{pH}=7.4$. Aliquots of $1 \mathrm{~mL}$ were taken at the indicated time points $(0.5,1,3,6,24,48,72,96,144,168,240,312,408$ hours after bead formation) over 17 days, and replaced with $1 \mathrm{~mL}$ of fresh medium. Quantification of the amount of ketoprofen in the aliquots was performed by UHPLC-ESI-HRMS analysis, following a protocol adapted from previous work. ${ }^{[42]}$ For detailed procedure, see Supplementary Material S-13.

\section{Supplementary Material}

Supporting information for this article is available on the WWW under http:

\section{Acknowledgements}

The authors acknowledge the financial support from the Swiss National Science Foundation (Grant $\left.N^{\circ} 310030 \mathrm{E}-164250\right)$ and the Foundation Insuleman. The authors thank Dr Aurélien Bornet for his assistance with NMR experiments and Dr Daniel Ortiz for the quantification of ketoprofen release by HPLCMS.

\section{Author Contribution Statement}

The manuscript was written through contributions of all authors. LS synthesized the Alg-PEG derivatives, and designed, produced and characterized the multicomponent MS. FN contributed to the design and preparation of drug eluting MS. SGL designed the experiments and supervised the work. LS and SGL prepared the manuscript.

\section{References}

[1] M. A. Fischbach, J. A. Bluestone, W. A. Lim, 'Cell-based therapeutics: The next pillar of medicine', Sci. Transl. Med. 2013, 5, 1-7.

[2] T. RJ. Heathman, A. W. Nienow, M, J. McCall, K. Coopman, B. Kara, C. J. Hewitt, ' The translation of cell-based therapies: Clinical landscape and manufacturing challenges', Regen. Med. 2015, 10, 49-64.

[3] E. Anassi, U. A. Ndefo, 'Sipuleucel-T (Provenge) injection the first immunotherapy agent (Vaccine) for hormone-refractory prostate cancer', Pharm. Ther. 2011, 36, 197202.

[4] P. P. Zheng, J. M. Kros, J, Li, 'Approved CAR T cell therapies: ice bucket challenges on glaring safety risks and long-term impacts', Drug Discov. Today 2018, 23, 11751182

[5] M. T. Chang, 'Semipermeable Microcapsules', Science 1964, 146, 524-525.

[6] R. Mahou, S. Passemard, M. Carvello, A. Petrelli, F. Noverraz, S. Gerber-Lemaire, C. Wandrey, 'Contribution of polymeric materials to progress in xenotransplantation of microencapsulated cells: a review', Xenotransplantation 2016, 23, 179-201.

[7] D. A. Foyt, M. D. A. Norman, T. T. L. Yu, E. Gentleman, 'Exploiting Advanced Hydrogel Technologies to Address Key Challenges in Regenerative Medicine', Adv. Healthc. Mater. 2018, 7, 1700939.

[8] N. Mitrousis, A. Fokina, M. S. Shoichet, 'Biomaterials for cell transplantation', Nat. Rev. Mater. 2018, 3, 441-456.

[9] T. Vermonden, B. Klumperman, 'The past, present and future of hydrogels', Eur. Polym. J. 2015, 72, 341-343.

[10] P. Sánchez, R. M. Hernández, J. L. Pedraz, G. Orive, 'Encapsulation of Cells in Alginate Gels', in: Guisan J.M. (eds) Immobilization of Enzymes and Cells. Methods in Biotechnology ${ }^{T M}, \mathbf{2 0 1 3}, 22$, Humana Press. 
[11] L. Szabo, S. Gerber-lemaire, C. Wandrey, 'Strategies to Functionalize the Anionic Biopolymer Na-Alginate without Restricting Its Polyelectrolyte Properties', Polymers 2020, 12, 919 .

[12] B. E. Tuch, G. W. Keogh, L. J. Williams, W. Wu, J. L. Foster, V. Vaithilingam, R. Philips, 'Safety and Viability of Microencapsulated Human Islets Transplanted into Diabetic Humans', Diabetes Care 2009, 32, 1887-1889.

[13] D. Jacobs-Tulleneers-Thevissen, M. Chintinne, Z. Ling, P. Gillard, L. Schoonjans, G. Delvaux, B. L. Strand, F. Gorus, B. Keymeulen, D. Pipeleers, 'Sustained function of alginate-encapsulated human islet cell implants in the peritoneal cavity of mice leading to a pilot study in a type 1 diabetic patient', Diabetologia $2013,56,1605-1614$.

[14] D. W. Scharp, P. E. Lacy, J. V. Santiago, C. S. McCullough, L. G. Weide, L. Falqui, P. Marchetti, R. L. Gingerich, A. S. Jaffe, P. E. Cryer, C. B. Anderson, M. W. Flye, 'Insulin independence after islet transplantation into type I diabetic patient', Diabetes 1990, 39, 515-518.

[15] R. Calafiore, G. Basta, G. Luca, A. Lemmi, M. P. Montanucci, G. Calabrese, L. Racanicchi, F. Mancuso, P. Brunetti, 'Microencapsulated pancreatic islet allografts into nonimmunosuppressed patients with type 1 diabetes', Diabetes Care 2006, 29, 137-138.

[16] G. Basta, P. Montanucci, G. Luca, C. Boselli, G. Noya, B. Barbaro, M. Qi, K. P. Kinzer, J. Oberholzer, R. Calafiore, 'Long-term metabolic and immunological follow-up of nonimmunosuppressed patients with type 1 diabetes treated with microencapsulated islet allografts: Four cases', Diabetes Care 2011, 34, 2406-2409.

[17] R. B. Elliott, L. Escobar, P. L. J. Tan, M. Muzina, S. Zwain, C. Buchanan, 'Live encapsulated porcine islets from a type 1 diabetic patient 9.5 yr after xenotransplantation', Xenotransplantation 2007, 14, 157-161.

[18] S. Ponce, G. Orive, R. Hernández, A. R. Gascón, J. L. Pedraz, B. J. de Haan, M. M. Faas, H. J. Mathieu, P. de Vos 'Chemistry and the biological response against immunoisolating alginate-polycation capsules of different composition', Biomaterials 2006, 27, 4831-4839.

[19] S. K. Tam, S. Bilodeau, J. Dusseault, G. Langlois, J.-P. Hallé, L. H. Yahia, 'Biocompatibility and physicochemical characteristics of alginate-polycation microcapsules', Acta Biomaterialia 2011, 7, 1683-1692.

[20] C. García-Astrain, L. Avérous, 'Synthesis and evaluation of functional alginate hydrogels based on click chemistry for drug delivery applications', Carbohydrate Polymers 2018, 190, 271-280.

[21] M. H. Ghanian, H. Mirzadeh, H. Baharvand, 'In Situ Forming, Cytocompatible, and Self-Recoverable Tough Hydrogels Based on Dual lonic and Click Cross-Linked Alginate', Biomacromolecules 2018, 19, 1646-1662.

[22] R. M. Desai, S. T. Koshy, S. A. Hilderbrand, D. J. Mooney, N. S. Joshi, 'Versatile click alginate hydrogels crosslinked via tetrazine-norbornene chemistry', Biomaterials $2015,15,30-37$.

[23] J. C. Breger, B. Fisher, R. Samy, S. Pollack, N. S. Wang, I. Isayeva, 'Synthesis of 'click' alginate hydrogel capsules and comparison of their stability, water swelling, and diffusion properties with that of $\mathrm{Ca}^{+2}$ crosslinked alginate capsules', J. Biomed. Mater. Res. B Appl. Biomater. 2015, 103, $1120-1132$.

[24] R. Mahou, N. M. Tran, M. Dufresne, C. Legallais, C. Wandrey, 'Encapsulation of Huh-7 cells within alginate-poly(ethylene glycol) hybrid microspheres', J. Mater. Sci: Mater. Med. 2012, 23, 171-179.

[25] R. Mahou, F. Borcard, V. Crivelli, E. Montanari, S. Passemard, F. Noverraz, S. Gerber-Lemaire, L. H. Bühler, C. Wandrey, 'Tuning the Properties of Hydrogel Microspheres by Adding Chemical Cross-linking Functionality to Sodium Alginate', Chem. Mater. 2015, 27, 4380-4389.

[26] S. Passemard, L. Szabó, F. Noverraz, E. Montanari, C. Gonelle-Gispert, L. H. Bühler, C. Wandrey, S. Gerber-Lemaire, 'Synthesis Strategies to Extend the Variety of Alginate-Based Hybrid Hydrogels for Cell Microencapsulation', Biomacromolecules 2017, 18, 2747-2755.

[27] J. M. Anderson, A. Rodriguez, D. T. Chang, 'Foreign body reaction to biomaterials', Seminars in Immunology 2008, 20, 86-100.

[28] A. S. Sawhney, J. A. Hubbell, 'Poly(ethylene oxide)-graft-poly(L-lysine) copolymers to enhance the biocompatibility of poly(L-lysine)-alginate microcapsule membranes', Biomaterials 1992, 13, 863-870.

[29] J. N. Zheng, H. G. Xie, W. T. Yu, X. D. Liu, W. Y. Xie, J. Zhu, X. J. Ma, 'Chitosan-g-MPEG-modified alginate/chitosan hydrogel microcapsules: A quantitative study of the effect of polymer architecture on the resistance to protein adsorption', Langmuir 2010, 26, 17156-17164.

[30] L. Moroni, M. Klein Gunnewiek, E. M. Benetti, 'Polymer brush coatings regulating cell behavior: Passive interfaces turn into active', Acta Biomaterialia 2014, 10, 23672378 .

[31] M. Spasojevic, S. Bhujbal, G. Paredes, B. J. de Haan, A. J. Schouten, P. de Vos, 'Considerations in binding diblock copolymers on hydrophilic alginate beads for providing an immunoprotective membrane', J. Biomed. Mater. Res. A 2014, 102, 1887-1896.

[32] V. Vaithilingam, M. D. M. Evans, A. Rowe, P. A. Bean, B. E. Tuch, 'Coencapsulation of target effector cells with mesenchymal stem cells reduces pericapsular fibrosis and improves graft survival in a xenotransplanted animal model', Cell Transplantation 2016, 25, 1299-1317.

[33] V. Vaithilingam, M. D. M. Evans, D. M. Lewy, P. A. Bean, S. Bal, B. E. Tuch, 'Co-encapsulation and co-transplantation of mesenchymal stem cells reduces pericapsular fibrosis and improves encapsulated islet survival and function when allografted', Sci. Rep. 2017, 7, 1-13.

[34] E. Vågesjö, G. Christoffersson, T. B. Waldén, P.-O. Carlsson, M. Essand, O. Korsgren, M. Phillipson, 'Immunological shielding by induced recruitment of regulatory TLymphocytes delays rejection of islets transplanted in muscle', Cell Transplantation 2015, 24, 263-276.

[35] M. Gliwiński, D. Iwaszkiewicz-Grześ, P. Trzonkowski, 'Cell-Based Therapies with T Regulatory Cells', BioDrugs $2017,31,335-347$.

[36] B. Layek, S. Mandal, 'Natural polysaccharides for controlled delivery of oral therapeutics: a recent update', Carbohydrate Polymers 2020, $230,115617$.

[37] T. T. Dang, A. V. Thai, J. Cohen, J. E. Slosberg, K. Siniakowicz, J. Doloff, M. Ma, J. Hollister-Lock, K. M. Tang, Z. Gu, H. Cheng, G. C. Weir, R. Langer, D. A. Anderson, 'Enhanced function of immuno-isolated islets in diabetes therapy by co-encapsulation with an anti-inflammatory drug', Biomaterials 2013, 34, 5792-5801. 


\section{HELVETICA}

[38] S. A. Azadi, E. Vasheghani-Farahani, S. Hashemi-Najafbabadi, A. Godini, 'Co-encapsulation of pancreatic islets and pentoxifylline in alginate-based microcapsules with enhanced immunosuppressive effects', Prog. Biomater. 2016, 5, 101-109.

[39] M. Ricci, P. Blasi, S. Giovagnoli, C. Rossi, G. Macchiarulo, G. Luca, G. Basta, R. Calafiore, 'Ketoprofen controlled release from composite microcapsules for cell encapsulation: Effect on post-transplant acute inflammation', J. Control. Release 2005, 107, 395-407.

[40] L. A. Omtvedt, M. Ø. Dalheim, T. T. Nielsen, K. L. Larsen, B. L. Strand, F. L. Aachmann, 'Efficient Grafting of Cyclodextrin to Alginate and Performance of the Hydrogel for Release of Model Drug', Sci. Rep. 2019, 9, 1-11.

[41] L. Szabó, C. Gonelle-Gispert, E. Montanari, F. Noverraz, A. Bornet, L. H. Bühler, S. Gerber-Lemaire, 'Cross-Reactive Alginate Derivatives for the Production of Dual Ionic-Covalent Hydrogel Microspheres Presenting Tunable Properties for Cell Microencapsulation', ACS Appl. Polym. Mater. 2019, 1, 1326-1333.

[42] F. Noverraz, E. Montanari, J. Pimenta, L. Szabó, D. Ortiz, C. Gonelle-Gispert, L. H. Bühler, S. Gerber-Lemaire, 'Antifibrotic Effect of Ketoprofen-Grafted Alginate Microcapsules in the Transplantation of Insulin Producing Cells', Bioconjug. Chem. 2018, 29, 1932-1941.

[43] J. L. Drury, R. G. Dennis, D. J. Mooney, 'The tensile properties of alginate hydrogels', Biomaterials 2004, 25, 3187-3199.

[44] B. Thu, P. Bruheim, T. Espevik, O. Smidsrød, P. Soon-Shiong, G. Skjåk-Bræk, 'Alginate polycation microcapsules: II. Some functional properties', Biomaterials 1996, 17, 1069-1079.

[45] J. Lakey, E. Botvinick, A. Najdahmadi, 'Diffusion coefficient of alginate microcapsules used in pancreatic islet transplantation, a method to cure type 1 diabetes', Nanoscale Imaging, Sensing, Actuation Biomed. Appl. XV. Int. Soc. Opt. Photonics 2018, 10506.

[46] A. Najdahmadi, J. R. Lakey, E. Botvinick, 'Structural Characteristics and Diffusion Coefficient of Alginate Hydrogels Used for Cell Based Drug Delivery', MRS Adv. 2018, $3,2399-2408$. 


\section{Entry for the Table of Contents}
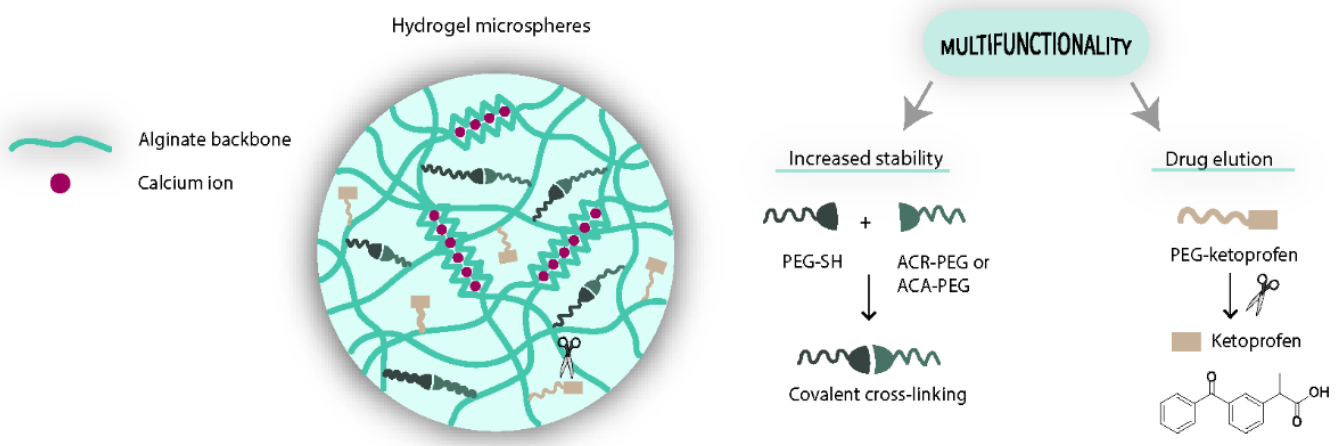

\section{Twitter}

Functionalization of sodium alginate with cross-reactive and drug-conjugated PEG derivatives allows the formation of multicomponent hydrogel microspheres presenting enhanced performance for cell encapsulation applications. 